\title{
STUDY OF LONGITUDINAL SPACE-CHARGE WAVE DYNAMICS IN SPACE-CHARGE DOMINATED BEAMS*
}

\author{
K. Tian", Y. Zou, Y. Cui, Y. Huo, M. Reiser, I. Haber, R. Kishek, and P. G. O’Shea \\ Institute for Research in Electronics and Applied Physics, University of Maryland, \\ College Park, MD 20742
}

\begin{abstract}
Understanding the dynamics of longitudinal spacecharge waves is very important for advanced accelerator research. Although analytical solutions of space-charge wave equations based on the cold fluid model exist in one dimension, there are few results for two-dimensional wave evolution. One-dimensional theory predicts two eigen solutions, given an initial perturbation. One is called the fast wave, which moves toward the beam head in the beam frame and the other is termed the slow wave, which moves backward in the beam frame. In this paper, we report experimental results of space charge wave studies conducted on a 2.3 meter long straight beam line at the University of Maryland. An energy analyzer is used to directly measure the energy of space-charge waves at the end of the transport line, which demonstrates the decomposition of an initial current perturbation into a slow wave and a fast wave. A PIC code, WARP [1], is used to simulate this experiment and the behavior of longitudinal waves in space-charge dominated beams in an R-Z geometry. Simulations shown here also demonstrate if the initial current and velocity perturbation strengths are chosen properly, only fast or slow waves could be selectively generated.
\end{abstract}

\section{INTRODUCTION}

Much interest has recently been generated in intense beams for applications such as High-Energy-Density Physics (HEDP), Heavy Ion Fusion (HIF), and the developments of the Spallation Neutron Source (SNS) and free electron lasers (FEL). Successful operation of such machines requires more detailed knowledge and understanding of the physics of the high intensity beams in which space-charge forces play a much more important role than in conventional accelerators. The longitudinal space-charge waves can be generated by density perturbations or energy perturbations caused by many factors, such as the discontinuity of the beam transportation modules, fluctuations in the bunch, or the mismatch of the focusing channels. These perturbations tend to excite longitudinal instabilities in the beam upon interacting with the external transport and acceleration environments. Energy dispersion caused by the energy modulations of space-charge waves will also introduce troubles in some high intensity machines.

Theoretical investigations on the evolutions of spacecharge waves have been carried out since 1980s [2]. The

\footnotetext{
*Work supported by the U.S. Department of Energy

grant numbers: DE-FG02-94ER40855 and DE-FG02-92ER54178

\#kaitian@umd.edu
}

most important analysis of the space-charge waves is the linear theory based on a one-dimensional cold fluid theory. In order to study the evolution of the space-charge waves, a special initial condition is used to solve the onedimensional cold fluid equations [3]. $I, v$ and $\Lambda$ are the current, velocity and line charge density of the beam, the subscript 0 represents the unperturbed values and the subscript 1 represents the perturbed values. The initial conditions and boundary conditions are: (a) There is no perturbation anywhere along the $\mathrm{z}$-axis when $\mathrm{t}<0$. (b) At $\mathrm{z}=0$ for $\mathrm{t}>0+\mathrm{a}$ localized velocity perturbation and current perturbation are introduced in the form:

$$
\begin{aligned}
& v_{1}(0, t)=\delta v_{0} h(t) \\
& I_{1}(0, t)=\eta I_{0} h(t)
\end{aligned}
$$

$\delta$ is a small, positive quantity to specify the strength of the velocity perturbation. $\eta$ is a small quantity to specify the strength of the initial current perturbation and can be negative if the velocity increase causes a current decrease. $\mathrm{h}(\mathrm{t})$ is any smooth function with amplitude of unity, which represents the shape of the perturbation and is supposed to vanish when $t$ is equal or smaller than zero. Thus, with the omission of higher order term, the line charge density perturbation can be expressed as:

$$
\Lambda_{1}(0, t)=(\eta-\delta) \Lambda_{0} h(t)
$$

The solutions of the linearized continuity and momentum transfer equations are:

$$
\begin{aligned}
& \Lambda_{1}(z, t)=-0.5 \Lambda_{0}[\delta V-(\eta-\delta)] h\left(t-t_{s}\right) \\
& +0.5 \Lambda_{0}[\delta V+(\eta-\delta)] h\left(t-t_{f}\right) \\
& v_{1}(z, t)=0.5 v_{0}[\delta-(\eta-\delta) / V] h\left(t-t_{s}\right) \\
& +0.5 v_{0}[\delta+(\eta-\delta) / V] h\left(t-t_{f}\right) \\
& I_{1}(z, t)=-0.5 I_{0}[\delta V-\eta+(\eta-\delta) / V] h\left(t-t_{s}\right) \\
& +0.5 I_{0}[\delta V+\eta+(\eta-\delta) / V] h\left(t-t_{f}\right)
\end{aligned}
$$

Here, $\mathrm{V}=\mathrm{v}_{0} / \mathrm{c}_{\mathrm{s}} ; \mathrm{t}_{\mathrm{s}}=\mathrm{z} /\left(\mathrm{v}_{0}-\mathrm{c}_{\mathrm{s}}\right) ; \mathrm{t}_{\mathrm{f}}=\mathrm{z} /\left(\mathrm{v}_{0}+\mathrm{c}_{\mathrm{s}}\right) ; \mathrm{c}_{\mathrm{s}}$ is called sound speed, which is the velocity of space-charge waves in the beam frame, in analogy to the propagation of sound waves in a gas. All these expressions have two terms: the first term is the slow wave; the second term is the fast wave. Both the fast wave and the slow wave keep the shape of the initial perturbation, while the amplitude and polarity are decided by the initial conditions.

It is very easy to see that the condition for generating only the fast wave is:

$$
\frac{\eta}{\delta}=1+\frac{v_{0}}{c_{s}}
$$

The condition for generating a pure slow wave is:

$$
\frac{\eta}{\delta}=1-\frac{v_{0}}{c_{s}}
$$




\section{SIMULATIONS IN WARP}

Although pure fast or slow waves were created in experiment in 1990s [3], there is no related simulation results published yet. In this section, the R-Z geometry module in WARP is used to generate a pure fast wave.

The WARP simulation uses typical beam parameters from the Long Solenoid Experiment (LSE) [4] at the University of Maryland. We use a beam pulse of $100 \mathrm{~ns}$, a main beam kinetic energy $E_{0}=5090 \mathrm{eV}$, an unperturbed beam current $I_{0}=100 \mathrm{~mA}$, a beam radius $a=0.5 \mathrm{~cm}$, and a beam pipe radius $b=1.905 \mathrm{~cm}$. Additional beam parameters can be derived from the above: e.g., main beam velocity $v_{0}=4.2314 \times 10^{7} \mathrm{~m} / \mathrm{s}$; generalized perveance $K=0.004049$; and the sound speed of space-charge wave $c_{s}=3.083 \times 10^{6} \mathrm{~m} / \mathrm{s}$. For these parameters, from equation (5) and (6), the condition for generating pure fast wave is $\eta / \delta=14.725$.

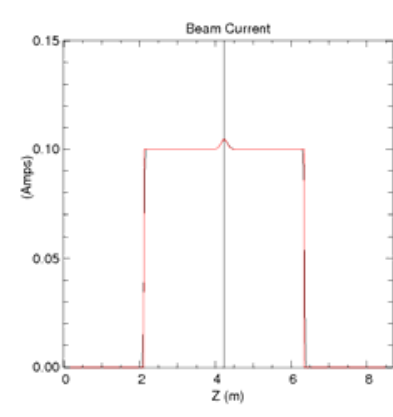

(a) Initial current

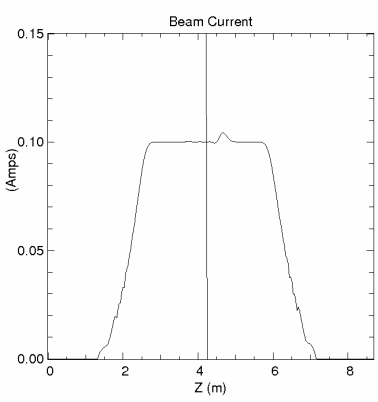

(c) Current after $6 \mathrm{~m}$

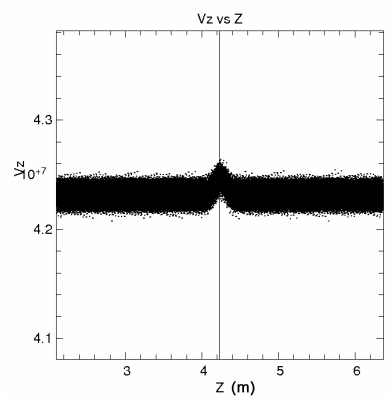

(b) Initial velocity

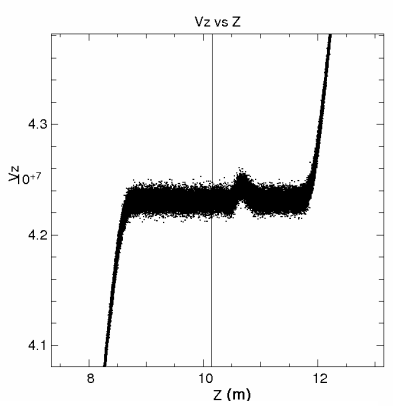

(d) Velocity after $6 \mathrm{~m}$ Figure 1: The WARP simulation for generating a pure fast wave.

In order to generate a single fast wave in WARP, an initial current perturbation with a strength $\eta=0.04417$ and an initial velocity perturbation with a strength $\delta=0.003$ are loaded to the beam. Both of these perturbations are $20 \mathrm{~ns}$ wide and located at the beam center with a Gaussian shape. A uniform focusing channel is imposed to keep the $100 \mathrm{~mA}$ beam matched at $0.5 \mathrm{~cm}$ radius. Other numerical settings for these two cases in WARP are: 128 cells in R direction, 256 cells in $\mathrm{z}$ direction, and 500000 macro particles.

The initial current profile in the beam frame and initial velocity in the lab frame are shown in Figures 1(a) and (b). After propagating 6 meters in the lab frame, the corresponding current profile and velocity profile are shown in Figure 1(c) and Figure 1(d) respectively. The vertical lines in all the figures mark the approximate position of the beam center, where the perturbation was initially launched. One can find that both the current perturbations and the velocity perturbations keep their initial shapes and move toward the beam head. A similar simulation, with the appropriate parameters, was able to generate a single slow wave in the code.

\section{EXPERIMENT}

Equations (4.a) to (4.c) infer that when a pure initial density perturbation is generated, i.e. $\eta$ is not zero and $\delta$ is zero, energy modulations can be excited due to the conversion from initial density modulations. In this section, a high-resolution energy analyzer is used to observe this phenomenon in the LSE.

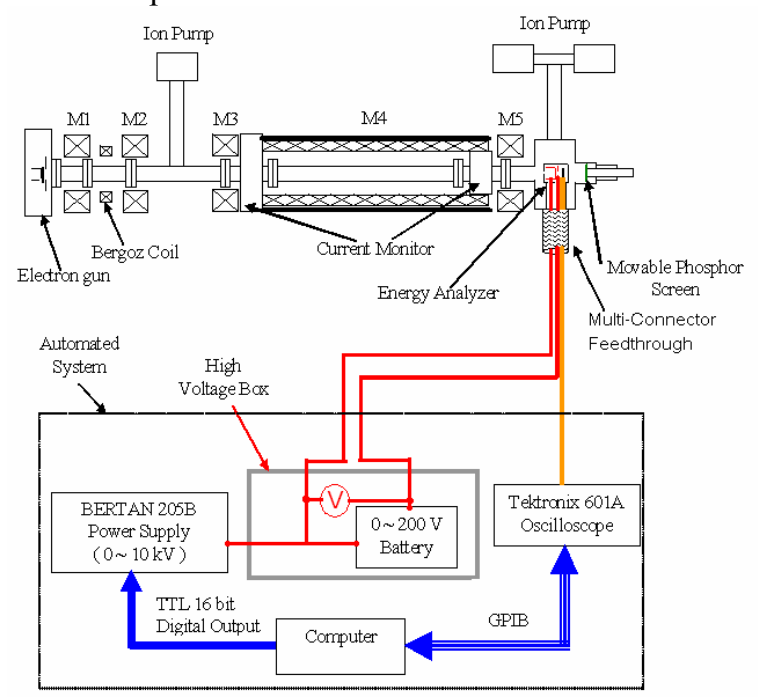

Figure 2: Schematics of the LSE system

\section{Experimental System}

The schematics of the experimental system are shown in Figure 2. A thermionic triode gun is used as the electron beams source. A high-resolution energy analyzer is installed in a diagnostic chamber at the end of the transportation line. There are five solenoids in the whole system. The long solenoid M4, which is 1.4 meter long, serves as a uniform focusing channel to transport the beam into the energy analyzer. In order to match the beam into the long solenoid, three short solenoids, M1, M2 and M3, are placed in the system. Another short solenoid M5 is placed between the exit of the long solenoid and the energy analyzer in order to control the amount of current injected into the energy analyzer.

One Bergoz fast current transformer is located between solenoids M3 and M4, and is about $20 \mathrm{~cm}$ from the aperture of the gun. Four ion pumps maintain a high vacuum. The first ion pump is located at the electron gun with a capacity of $8 \mathrm{l} / \mathrm{s}$. The other three ion pumps have capacities of $40 \mathrm{l} / \mathrm{s}$. One is located between the second and third solenoids and two are located at the diagnostic 
chamber. The beam pipeline is connected with an automated measurement system including a high-voltage power supply, a Tektronix oscilloscope (TEK DSA 601A) and a computer.

\section{Generation of Initial Current Perturbations}

The basic idea of this experiment is to observe the energy perturbation, which is excited by a pure current perturbation. In order to produce a pure current modulation at the electron gun, we need to change the working condition of the electron gun. The beam pulse of this electron gun is created by a cathode pulse, which is generated by a pulse generation cable [5]. In the experiment, a cable is connected to the middle of the pulse generation transmission line through a " $T$ " connector. As a result, a perturbation is generated at the center of the cathode pulse, which can introduce a localized perturbation to the beam current. The beam energy in the experiment is $5 \mathrm{KeV}$, while the perturbation of the cathode pulse is very small compared to this value, so the energy modulation created at the gun can be neglected. Using a laser to impinge the photocathode can generate pure initial current perturbations [6-7]; we may use this method in the future experiment.

Figure 3 shows the current profile with perturbations measured by the Bergoz current monitor in the system. The main beam current is $77.4 \mathrm{~mA}$, the current perturbation is $10.85 \mathrm{~mA}$.

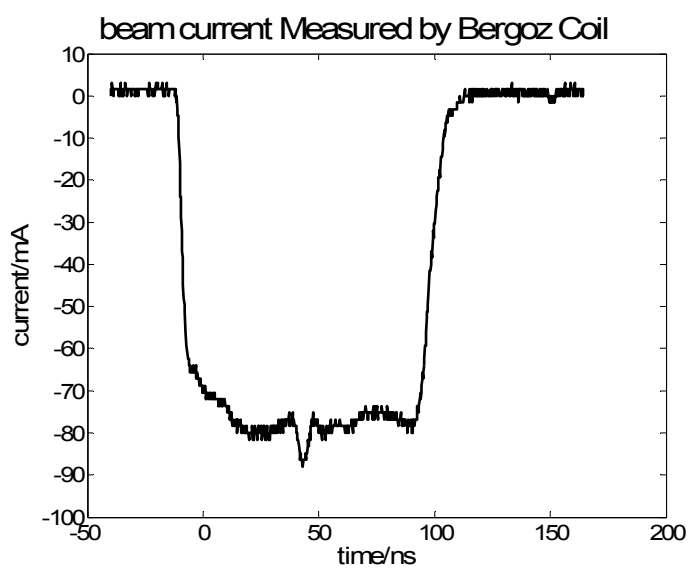

Figure 3: Current profile measured by Bergoz Coil

According to the theoretical predictions, both current and velocity perturbations will exist in the form of a fast wave and a slow wave at the downstream of the beamline. The energy perturbation waveform can be measured by the Energy analyzer after the long solenoid.

\section{Measurement of Energy Perturbations}

With the retarding voltage energy analyzer [8], located 2.3 meters before the aperture of the electron gun, the energy spectrum can be obtained for different locations along the beam pulse. The integration of the energy spectrum at a specific location is the mean energy of that point. Therefore, the mean energy waveform can be plotted in Figure 4.

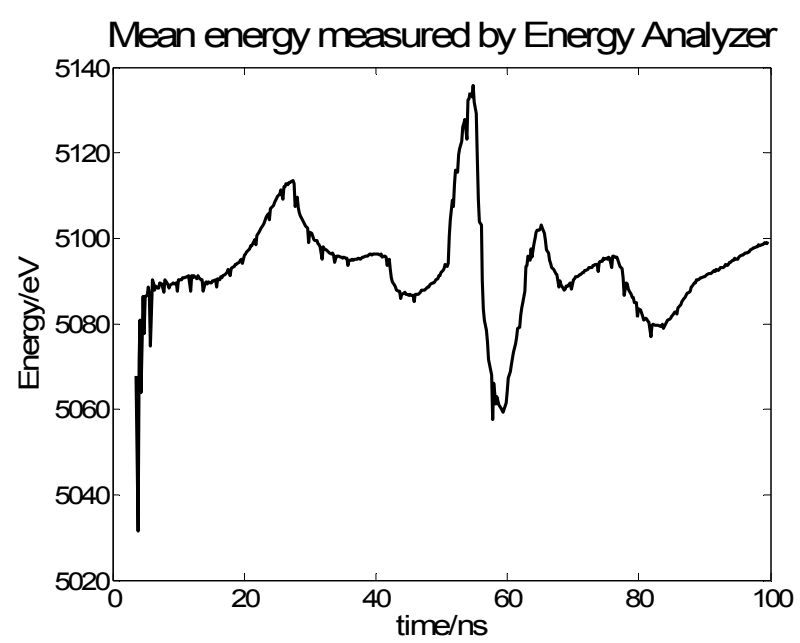

Figure 4: Mean energy waveforms measured derived from energy analyzer signal

As the theory predicts, with the initial condition of pure density modulation, energy modulation can be achieved. The energy modulation splits into two peaks at the beam center: one is positive and near to the beam head, which corresponds to the fast wave, the other is a negative peak and near to the beam end, which corresponds to the slow wave. Some other peaks are obtained except for the peaks at the center. Compared with the initial currents in Figure 3, it is not difficult to find that they are due to the bumps near the beam head and beam end.

\section{CONCLUSION}

A 2-D WARP simulation has been carried out to generate a pure fast wave, the simulation result shows very good agreement with the one-dimensional linear theory. The energy modulation waveform converted from an initial density modulation is measured in a straight beam transportation system. The experimental result agrees well with the theoretical predictions.

\section{REFERENCES}

[1] D.P.Grote et al. Fusion Eng. Des 32-33, 1996, p.193200

[2] M. Reiser, Theory and Design of Charged Particle Beams, John Wiley \& Sons, Inc, New York, 1994

[3] J. G. Wang, et al, Physical Review Letters 71, 1993, p. 1836

[4] R. A. Kishek, invited talk, 15th International Symposium on Heavy Ion Inertial Fusion, NJ, 2004

[5] J. G. Wang et al, IEEE Transactions on Electron Devices. 37, 1990, p.2622.

[6] J. G. Neunann et al. Rev. Sci. Instrm. 75, 033303, 2004,

[7] Y. Huo, master thesis, University of Maryland, 2004

[8] Y. Cui et al, Rev. Sci. Instrm. 75, 2004, p.2738 